\title{
Genome-Wide Association Study of Loneliness Demonstrates a Role for Common Variation
}

\author{
Jianjun Gao ${ }^{1,2}$, Lea K Davis ${ }^{3}$, Amy B Hart ${ }^{4}$, Sandra Sanchez-Roige ${ }^{2}$, Lide Han', John T Cacioppo ${ }^{5}$ and \\ Abraham A Palmer*,1,2,6 \\ 'Department of Human Genetics, University of Chicago, Chicago, IL, USA; ${ }^{2}$ Department of Psychiatry, University of California, San Diego, La Jolla, \\ CA, USA; ${ }^{3}$ Vanderbilt Genetics Institute, Department of Medicine, Division of Genetic Medicine, Vanderbilt University Medical Center, Nashville, TN, \\ USA; ${ }^{4}$ Janssen R\&D, Spring House, PA, USA; ${ }^{5}$ Department of Psychology, University of Chicago, Chicago, IL, USA; ${ }^{6}$ Institute for Genomic Medicine, \\ University of California, San Diego, La Jolla, CA, USA
}

\begin{abstract}
Loneliness is a complex biological trait that has been associated with numerous negative health outcomes. The measurement and environmental determinants of loneliness are well understood, but its genetic basis is not. Previous studies have estimated the heritability of loneliness between 37 and 55\% using twins and family-based approaches, and have explored the role of specific candidate genes. We used genotypic and phenotypic data from 10760 individuals aged $\geqslant 50$ years that were collected by the Health and Retirement Study (HRS) to perform the first genome-wide association study of loneliness. No associations reached genome-wide significance $\left(p>5 \times 10^{-8}\right)$. Furthermore, none of the previously published associations between variants within candidate genes (BDNF, OXTR, RORA, GRM8, CHRNA4, IL-IA, CRHRI, MTHFR, DRD2, APOE) and loneliness were replicated ( $p>0.05)$, despite our much larger sample size. We estimated the chip heritability of loneliness and examined coheritability between loneliness and several personality and psychiatric traits. Our estimates of chip heritability (14-27\%) support a role for common genetic variation. We identified strong genetic correlations between loneliness, neuroticism, and a scale of 'depressive symptoms.' We also identified weaker evidence for coheritability with extraversion, schizophrenia, bipolar disorder, and major depressive disorder. We conclude that loneliness, as defined in this study, is a modestly heritable trait that has a highly polygenic genetic architecture. The coheritability between loneliness and neuroticism may reflect the role of negative affectivity that is common to both traits. Our results also reflect the value of studies that probe the common genetic basis of salutary social bonds and clinically defined psychiatric disorders.
\end{abstract}

Neuropsychopharmacology (2017) 42, 81 I-821; doi:I0.1038/npp.2016.197; published online 12 October 2016

\section{INTRODUCTION}

Humans are fundamentally social animals who form bonds with others for mutual aid and protection. For social species, the perception of being socially isolated even when in the presence of others signals danger and evokes a dysphoric state termed loneliness in humans (Cacioppo et al, 2014, 2015a). A variety of biological mechanisms have evolved that capitalize on aversive signals to motivate people to act in ways that are essential for reproduction and survival. Just as physical pain is an aversive signal that alerts us of potential tissue damage and motivates us to take care of our physical body, loneliness-triggered by a discrepancy between an individual's preferred and actual social relations-is part of a biological warning system that has evolved to alert us of threats or damage to our social body.

\footnotetext{
*Correspondence: Professor AA Palmer, Department of Psychiatry, University of California, San Diego, La Jolla, CA 92093, USA, Tel: + I 858534 2093, Fax: + I 858534 7653, E-mail: aap@ucsd.edu Received 20 April 2016; revised 29 August 2016; accepted 2 September 20 I6; accepted article preview online I5 September 2016
}

A substantial literature now shows that loneliness is a major risk factor for adverse physical (Holt-Lunstad et al, 2015) and mental (Cacioppo et al, 2015c) health outcomes. A recent meta-analysis of 70 independent prospective studies involving $>3$ million people who were followed for an average of 7 years shows that loneliness increases the odds of mortality by $26 \%$ even after controlling for objective social isolation and other potentially confounding factors (HoltLunstad et al, 2015). For instance, using longitudinal data from the Health and Retirement Study (HRS), Luo et al (2012) found that loneliness in 2002 predicted mortality over the subsequent 6 years even after controlling for demographic factors, health behaviors, and objective social isolation.

Investigations have found loneliness to be stable over years (see, eg, Boomsma et al, 2005) and to differ from other personality factors such as extraversion, neuroticism, depressive symptomatology shyness, and anxiety (see, eg, Cacioppo et al, 2006, 2010). Studies designed to identify the mechanisms underlying the association between loneliness and mortality have found that loneliness is associated with increased hypothalamic-pituitary-adrenocortical (HPA) 
activity (Adam et al, 2006; Cacioppo et al, 2006; Doane and Adam, 2010; Glaser et al, 1985; Kiecolt-Glaser et al, 1984; Steptoe et al, 2004), altered gene expression indicative of decreased inflammatory control and increased glucocorticoid insensitivity (Cole et al, 2007, 2011), increased inflammation, elevated vascular resistance, and blood pressure (Hackett et al, 2012; Hawkley et al, 2006, 2010b; Jaremka et al, 2013), higher rates of metabolic syndrome (Whisman, 2010), diminished immunity (Dixon et al, 2006; Glaser et al, 2005; Kiecolt-Glaser et al, 1984; Pressman et al, 2005; StraitsTröster et al, 1994), increased risk for age-related cognitive decline and dementia (Wilson et al, 2007), and increased sleep fragmentation (Cacioppo et al, 2002; Hawkley et al, 2010a; Jacobs et al, 2006; Kurina et al, 2011). Cross-lagged panel analyses have also shown that loneliness has also been associated with changes in psychological states that can contribute to morbidity and mortality, including increased depressive symptomatology (Booth, 2000; Cacioppo et al, 2006, 2010; VanderWeele et al, 2011), lower subjective wellbeing (Kong and You, 2013; VanderWeele et al, 2012), heightened vigilance for social threats (Cacioppo et al, 2015b), and decreased executive functioning (Baumeister and DeWall, 2005; Cacioppo et al, 2000; Hawkley et al, 2009).

The heritability of loneliness has been documented in twin and other studies using both children (Bartels et al, 2008; McGuire and Clifford, 2000) and adults (Boomsma et al, $2005,2006,2007)$. For instance, in an early longitudinal study of 8387 young adult and adult Dutch twins who participated in longitudinal surveys, Boomsma et al, (2005) analyzed variations in loneliness with genetic structural equation models. The estimate of genetic contributions to variation in loneliness in adults was $48 \%$, similar to the heritability estimates reported by McGuire and Clifford (2000) in their study of children. Boomsma et al (2005) found no evidence for sex or age differences in heritability. Subsequent twin studies have yielded heritability estimates ranging from 37 to 55\% (Boomsma et al, 2005, 2006; Distel et al, 2010; McGuire and Clifford, 2000; Waaktaar and Torgersen, 2012). Candidate gene studies for loneliness have concentrated primarily on systems related to monoamine neurotransmitters (eg, dopamine, serotonin) and other signaling pathways associated with human attachment (eg, oxytocin) (Goossens et al, 2015). Typical of candidate gene studies, they used modest sample sizes and therefore implicitly assumed relatively large effect sizes for the alleles being studied, a scenario that is inconsistent with the results of genome-wide association studies (GWAS) for numerous disease and nondisease traits (Hart et al, 2013).

In this study we have performed the first GWAS for loneliness. The UCLA loneliness scale is the most commonly used measure in the literature and has very good psychometric properties, including internal reliability, temporal stability, discriminant validity, convergent validity, construct validity, and predictive validity (Cacioppo et al, 2006; Russell, 1980, 1996). Importantly, the term 'loneliness' does not appear in this scale because respondents, especially males, have been found to be reluctant to report feeling lonely (Russell et al, 1980). Hence, the measurement of this phenotype is not dependent on the respondents' ability or willingness to self-report being lonely. Since 2002, the HRS has included a 3-item version of the UCLA loneliness scale that has also been shown to have very good psychometric properties, including internal reliability, concurrent validity (eg, $r=0.88$ with the full 20-item UCLA loneliness scale; Hughes et al, 2004), convergent and discriminant validity (Hughes et al, 2004), and predictive validity (eg, predicts mortality in the HRS sample over a 6-year period; Hughes et al, 2004; Luo et al, 2012). We used a genomic restricted maximum likelihood (GREML) method implemented in the Genetic Complex Trait Analysis (GCTA) software (Yang et al, 2011) to examine chip heritability that is specifically due to the additive effect of genotyped (or imputed) common variants. Loneliness and objective social isolation are often weakly correlated (Coyle and Dugan, 2012; Holt-Lunstad et al, 2015), although loneliness does tend to be lower in individuals who are married than those who are unmarried (eg, Hawkley et al, 2008). Analyses were therefore performed including marital status as a covariate. We also determined whether previously reported candidate gene associations could be replicated in the HRS. Finally, using polygenic risk scoring and estimates of genetic correlation, we were able to begin to probe possible shared genetic influences between personality traits and psychiatric diagnoses and loneliness.

\section{MATERIALS AND METHODS}

\section{Subjects}

The University of Michigan HRS is a longitudinal study that began in 1992 and includes more than 26000 Americans who are $>50$ years of age (Health_and_Retirement_Study, 2012). Our study included genotype data (both directly genotyped and imputed) obtained from dbGaP (accession number phs000428.v1.p1) on a total of 12454 subjects from HRS that were genotyped by the Center for Inherited Disease Research (CIDR). Permission to use the HRS data set was obtained through application to $\mathrm{dbGaP}$ by JTC. Phenotypic data were collected by the HRS on subjective experiences of loneliness during the 2006 and 2008 data collection waves.

\section{Loneliness Phenotype}

Loneliness was assessed using the 3-Item Leave Behind Questionnaire (LBQ) as part of a larger written questionnaire administered as part of the HRS (Hughes et al, 2004). Respondents were asked three questions. 'How often do you feel that you lack companionship? 'How often do you feel left out?' and 'How often do you feel isolated from others?' Possible answers were 'hardly ever, or never' (scored as 1); 'some of the time' (scored as 2); or 'often' (scored as 3 ). Thus, higher scores represent greater self-reported loneliness (Supplementary Figure S1). The total score on this 3-item loneliness scale has been shown to be highly correlated ( $r=0.88$ ) with the total score on the UCLA loneliness scale. Only participants who responded to all the three questions were included in our study. Pairwise correlation coefficients between questions were obtained using Spearman's correlation statistics in SAS version 9.3 (SAS Institute, Cary, NC).

We derived three phenotypes from the loneliness scale for subsequent genetic analyses: (1) 'linear'-a continuous phenotype obtained by summing the scores from all three questions, thus yielding a score between 3 (least lonely) and 9 (most lonely); (2) 'multivariate' - a single score for each question ranging from 1 (least lonely) to 3 (most lonely); and 
(3) 'case/control'-a dichotomous score in which participants who answered 1 on all three items were considered controls (totally loneliness score $=3$ ) and individuals with a loneliness score of $\geqslant 6$ were considered cases (participants with scores of 4 or 5 were treated as missing). There were nine subjects who answered the loneliness questions twice (first in 2006 and again in 2008); we used an average of their two scores. Frequency distributions by ancestry for the linear trait and case/control labels are shown in Supplementary Figure S2.

We considered sex, age (continuous), and marital status (categorical) as covariates in our analyses. Marital status was ascertained such that it consisted of six levels (married, annulled, separated, divorced, widowed, and never married). For the genetic analyses, we summarized these six levels into a binary variable (married or unmarried). Subjects for whom any of these three covariates were missing were excluded from all of our analyses.

\section{SNP Genotyping and Quality Control}

Genotyping of HRS subjects was performed by the NIH CIDR (http://www.cidr.jhmi.edu/), using the Illumina Human Omni-2.5 Quad BeadChip. Genotyping quality control was performed by the Genetics Coordinating Center at the University of Washington, Seattle, WA. Further information is available from the HRS website (http://hrsonline. isr.umich.edu/sitedocs/genetics/HRS_QC_REPORT_MAR2012. pdf). Additional more stringent QC was conducted for SNPbased heritability analyses, as models that include the joint effect of all SNPs are known to be sensitive to technical artifacts. For the directly genotyped data, we applied the recommended SNP quality filter provided by CIDR, yielding 1681327 SNPs. Quality control of the imputed genotype data was performed with the QCTOOL software package (http://www.well.ox.ac.uk/ gav/qctool/\#overview). SNPs with call rate $>95 \%$, MAF $>1 \%$, Hardy-Weinberg equilibrium $P>10^{-6}$, and an imputation info score $>0.5$ were retained for further analysis. Subject-level QC was performed with the GTOOL software package (http://www. well.ox.ac.uk/ cfreeman/software/gwas/gtool.html) and included iteratively removing one subject of any pair whose kinship coefficient was $>0.1$. The numbers of SNPs available after QC for each analysis are shown in Supplementary Table S1.

Because the participants in the HRS were a mixture of European Americans (EA, $n=7556)$, African Americans (AA, $n=1155$ ), and Hispanic Americans (HA, $n=695$ ), we calculated the first 10 principal components (PCs) from the genotype data to use as covariates. After manual inspection, we concluded that 1354 subjects did not clearly fit any of these categories (see Supplementary Figure S3) and were therefore excluded from both GREML and polygenic analyses.

\section{Genome-Wide Association Study}

We used Linear Mixed Model (LMM) or Multivariate Linear Mixed Models (MLMM) implemented in the Genome-wide Efficient Mixed Model Association (GEMMA) software package to further correct for residual population structure due to ancestry or cryptic relatedness in our GWAS (Zhou and Stephens, 2012). We examined the linear, multivariate, and case/control phenotype models using either directly genotyped or a combination of genotyped and imputed SNP data, adjusting for sex, age, and marital status (binary). We excluded SNPs with MAF $<0.01$. For the case/control studies, controls were coded as 0 and cases were coded as 1 , as suggested in the GEMMA documentation (Zhou and Stephens, 2012). The association analyses were performed using all 10760 subjects and separately in the subset of the 7556 EA subjects. We implemented the leave-one-chromosome-out (LOCO) method within the mixed model framework in order to avoid a loss of statistical power due to 'proximal contamination' or inclusion of the candidate marker (or markers in LD with the candidate marker) in the genetic relationship matrix (GRM) (Cheng et al, 2013; Yang et al, 2014).

\section{Analysis of Candidate Variants Identified in Prior Studies}

We identified 13 variants in 11 genes that have previously been associated with loneliness phenotypes in the published literature (Chou, 2010, 2014; Connelly et al, 2014; Lan et al, 2012; Lucht et al, 2009; Terracciano et al, 2010; Tsai et al, 2012; van Roekel et al, 2010, 2011, 2013; Verhagen et al, 2014; Wang et al, 2013). We examined the association of each of these SNPs with loneliness (linear, multivariate, and case/ control) in the results from the GWAS described above. For those candidate SNPs that were not directly genotyped or imputed in our study, we identified proxy SNPs with $r^{2}>0.8$ whenever possible. For SNPs that yielded $p<0.05$, we determined whether the direction of the association was consistent between the prior and current studies. We did not apply any correction for multiple comparisons.

\section{Estimation of Variance in Loneliness Explained by the Genotyped SNPs ('Chip Heritability')}

To estimate the proportion of phenotypic variance explained ('chip heritability'; $h_{\mathrm{g}}^{2}$ ), we used the GREML method implemented in GCTA (Yang et al, 2010). The purpose of the GREML method is to estimate the proportion of variation in a phenotype that is due to all SNPs. The GREML method is well established, has been described in detail, and exploits the fact that genotypic similarity (ie, 'relatedness', measured using genotyped SNPs) will be correlated with phenotypic similarity for phenotypes that are influenced by genetic variation. Additional individual-level quality control was implemented and distantly related individuals with pair-wise relationships were further filtered at two thresholds $\left(K_{\mathrm{IBS}}<0.05\right.$ and $\left.K_{\mathrm{IBS}}<0.025\right)$. Covariates included in the GREML analysis were age (continuous), self-reported sex (male/female), marital status (married/not married), and top 10 PCs. GREML analyses were run using only directly genotyped SNPs to construct the GRM. We obtained estimates of heritability for both the linear trait and the case/ control classification in the EA subset $(N=7556)$.

\section{Polygenic Risk Score (PRS) Analysis}

We investigated whether the genetic risk for loneliness overlaps with the genetic risk for several personality traits and psychiatric diseases (Supplementary Table S2). For each set ('discovery sample') of GWAS results (eg, SCZ2), we 
identified SNPs that were also genotyped in our HRS loneliness data ('target sample') and then used PLINK to LD-prune the SNPs $\left(r^{2}<0.2\right.$; using the '-indep-pairwise' command). The target sample was restricted to EAs to avoid confounding due to residual population stratification. SNPs with association $p$-values passing predetermined significance thresholds $\left(p<10^{-5}, 10^{-4}, 10^{-3}, 0.01,0.05,0.1,0.3\right.$, and 0.5 , respectively) in the discovery sample were extracted along with their risk alleles and odds ratios. For each significance threshold, a quantitative aggregate risk score was calculated for each EA individual in the target sample, defined as the sum of the number of risk alleles present at each locus weighted by the log of the odds ratio for that locus estimated from the discovery sample (as implemented in the PLINK 'score' command (Purcell et al, 2007). The relationship between aggregate risk score in relation to three phenotypes (eg, linear, multivariate, and case/control status) in the target sample was examined at each significance threshold using linear regression, multivariate regression, or logistic regression correspondingly.

\section{LD Score Regression (LDSC)}

To further investigate the genetic overlap between loneliness and various other traits (Supplementary Table S2), we used LDSC (Bulik-Sullivan et al, 2015a, b). We limited our analysis to the EA subjects and used the results from the case/control analysis shown in Figure 1. To standardize the input files, we followed quality controls as implemented by the LDSC python software package (https://github. com/bulik/ldsc). We used precalculated LD scores ('eur_w_ld_chr/' files; Finucane et al, 2015) for each SNP using individuals of European ancestry from the 1000 Genomes project that are suitable for LD score analysis in European populations. To restrict the analysis to wellimputed SNPs, the SNPs were filtered to HapMap3 SNPs (International Hapmap Consortium et al, 2010), and were required to have a MAF above 1\%. INDELs, structural variants, strand-ambiguous SNPs, and SNPs with extremely large effect sizes $\left(\chi^{2}>80\right)$ were removed.

\section{RESULTS}

\section{Demographics}

The distributions of responses to each question are shown in Supplementary Figure S1 and the sum of the three questions is shown in Supplementary Figure S2. Table 1 displays population characteristics according to loneliness status. Consistent with prior studies, loneliness was influenced by ancestry, decreased slightly but significantly with age, and did not differ by gender. In addition, consistent with prior studies, marital status had a large impact on our quantitative measure of loneliness, with all non-married categories showing greater loneliness compared with individuals who were married (Table 1).

\section{GWAS of Loneliness}

Figure 1 shows the results of our GWAS for the 7556 EA-only cohort using both quantile-quantile (QQ) and Manhattan plots. None of the GWAS yielded genome-wide significant associations $\left(p<5 \times 10^{-8}\right)$. The most significant results are listed in an Excel spreadsheet that is included in the Supplementary Material. We also performed these analyses using the full set of 10760 subjects, and the results were not meaningfully different (Supplementary Figures S4-S6).

\section{Previously Studied Candidate Genes}

Table 2 shows that we did not replicate any of the associations between loneliness and specific candidate genes that had been previously reported. None of these SNPs showed significant evidence for association $(p \leqslant 0.05$ without correction for multiple comparisons), with the exception of the gene MTHFR (Table 2), for which the direction of the association in our data was opposite to what was reported previously (Lan et al, 2012). Therefore, none of the previously reported associations could be replicated, despite our large sample size.

\section{Heritability Estimates for Loneliness}

We found that loneliness had a significant chip heritability (case/control 0.27, $\mathrm{SE}=0.12, p=0.01$; linear trait $\mathrm{PVE}=0.16$, $\mathrm{SE}=0.06, p=0.002$; Table 3). Because loneliness was significantly associated with self-reported ethnicity, we focused on the EA subset for heritability estimates to avoid confounding. To guard against any within-EA structure, we calculated heritability after adjusting for the top 10 PCs from the genotype data and also after additionally eliminating individuals with $K_{\mathrm{IBS}}>0.05$ and $K_{\mathrm{IBS}}>0.025$. Results were robust to these different approaches. Although the $h_{\mathrm{g}}^{2}$ estimate for the case/control phenotype was higher than the linear trait, the overlapping standard errors indicate that these estimates are not significantly different.

\section{Polygenic Score Analyses}

For these analyses, we used the 6924 distantly related/ unrelated EA subjects. Results for neuroticism (Table 4 and Supplementary Tables S3 and S4) showed unambiguously significant positive coheritability. We observed modest evidence for negative coheritability with extraversion (Supplementary Table S5); the multivariate analysis suggested that the questions 'How often do you feel that you lack companionship?' and 'How often do you feel left out?' showed the greatest coheritability with extraversion. We also observed modest evidence for negative coheritability with schizophrenia (SCZ1 and SCZ2, Supplementary Tables S6 and S7) and bipolar disorder (Supplementary Table S8); for these diseases the multivariate analysis suggested that the question 'How often do you feel left out?' showed the greatest coheritability. There was no evidence for coheritability with major depressive disorder (Supplementary Table S9) but a no-clinical trait called 'depressive symptoms' did show significant positive coheritability with loneliness (Supplementary Table S10); the multivariate analysis suggested that all three questions contributed to the observed coheritability.

\section{Genetic Correlation}

The results for LDSC analysis used the case/control loneliness GWAS summary statistics and produced results that were broadly similar to the results from the PRS. The genetic correlation between loneliness and personality traits was 
a

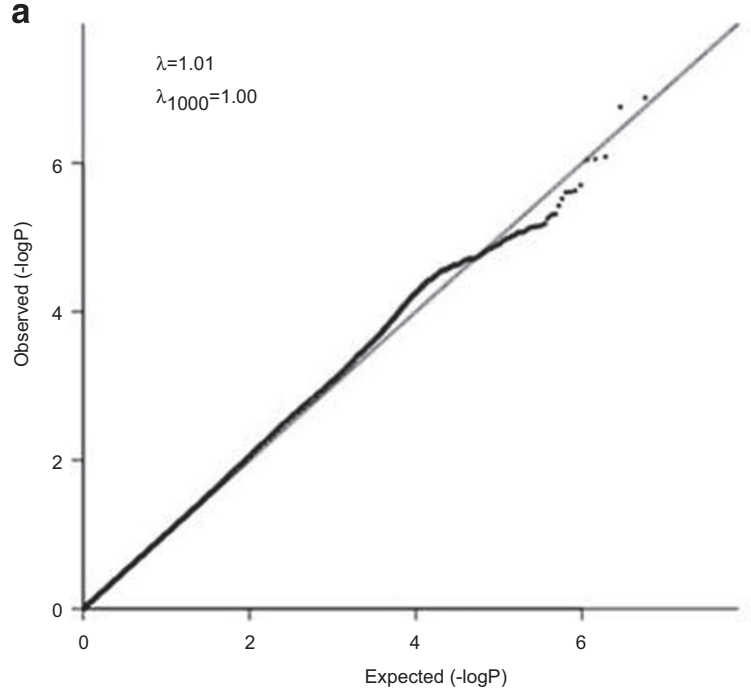

C

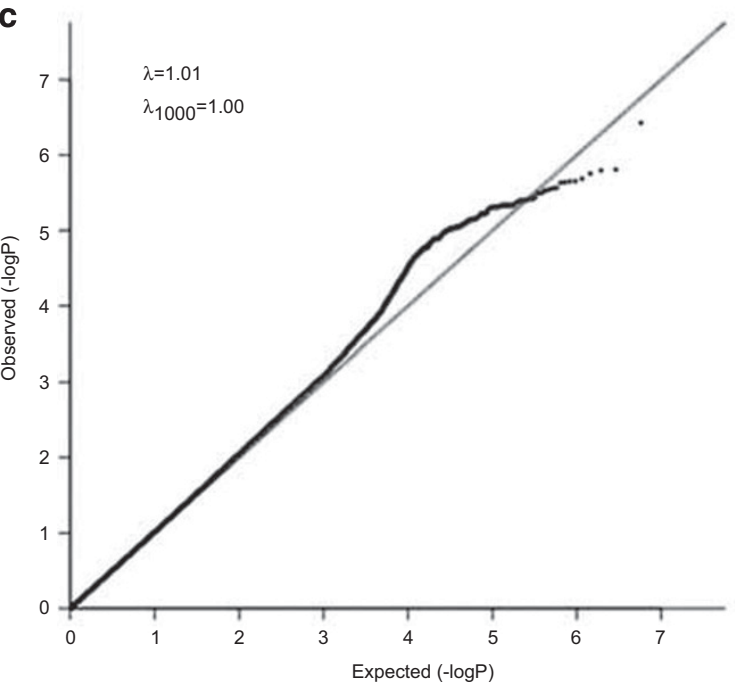

e

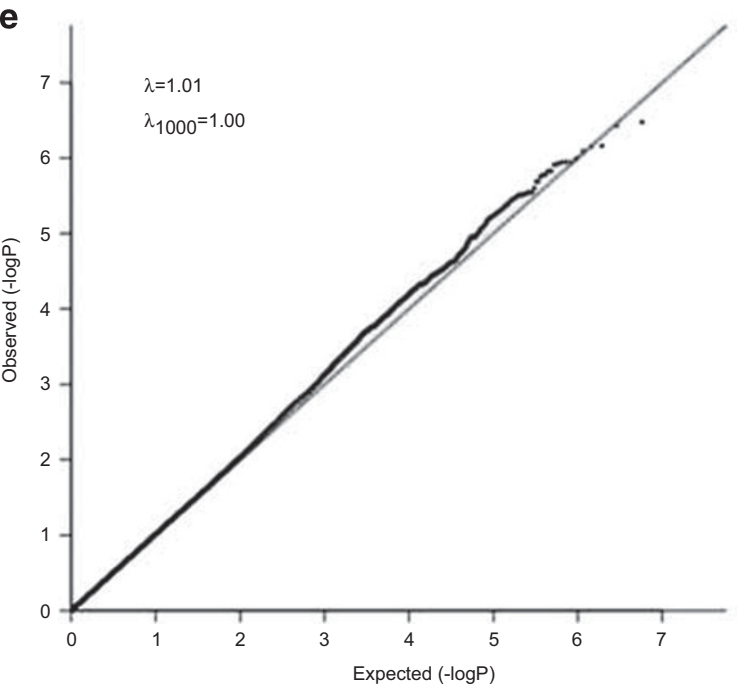

b

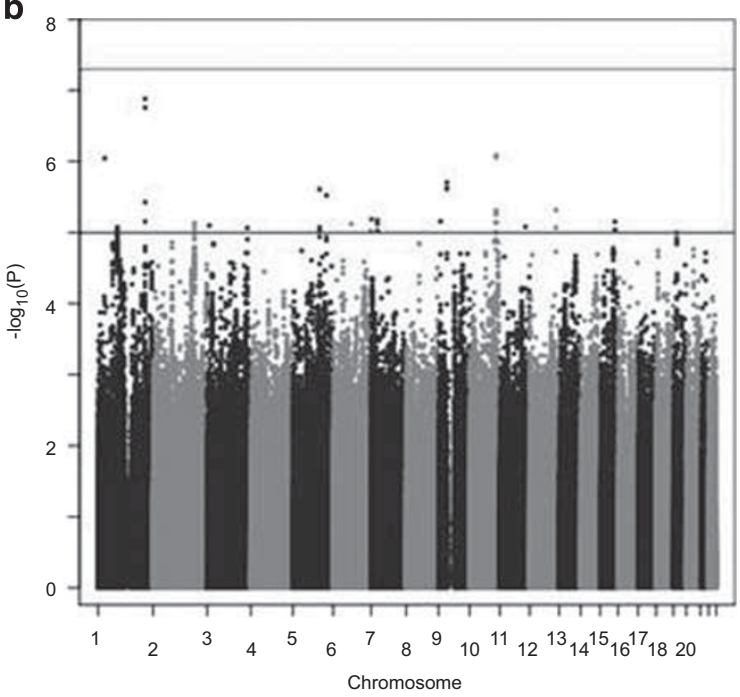

d

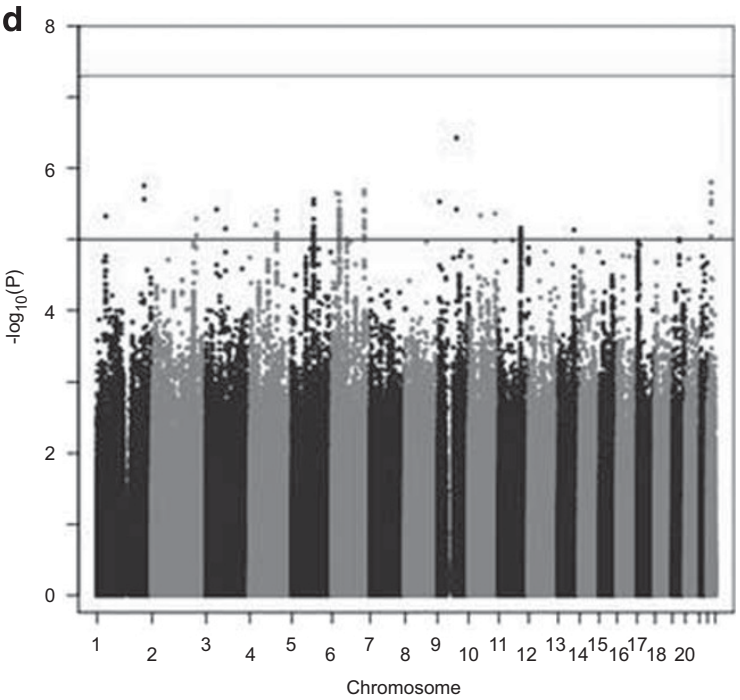

f

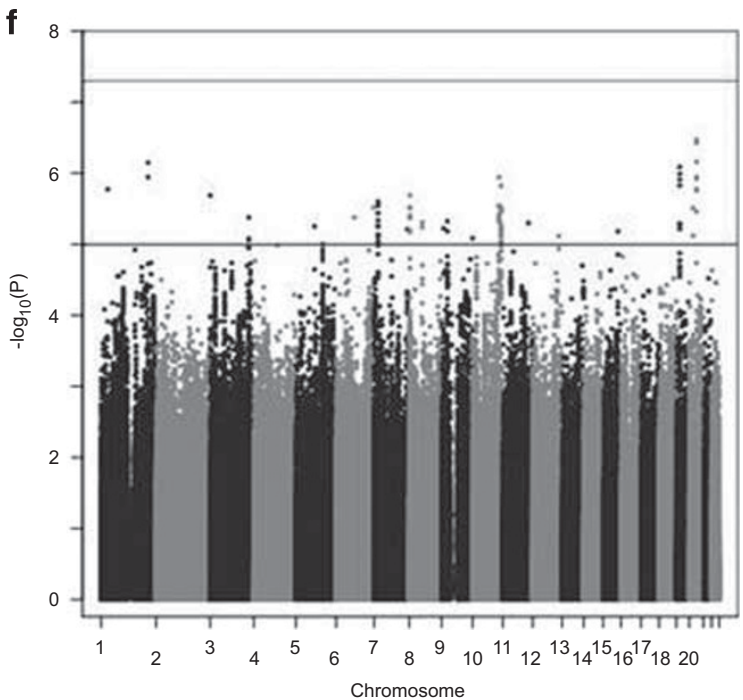

Figure I QQ and Manhattan plots of genome-wide association studies for loneliness in EA-only subjects using the imputed SNPs, calculated by mixed regression models with adjustments for age, gender, and marital status. (a, b) Linear mixed model $(n=7556)$; $(c, d)$ multivariate mixed model $(n=7556)$, and (e, f) case/control mixed model $(n=5228)$. 
Table I Participants' Characteristics of Health and Retirement Study (HRS) and Associations with Loneliness ${ }^{\mathrm{a}}$

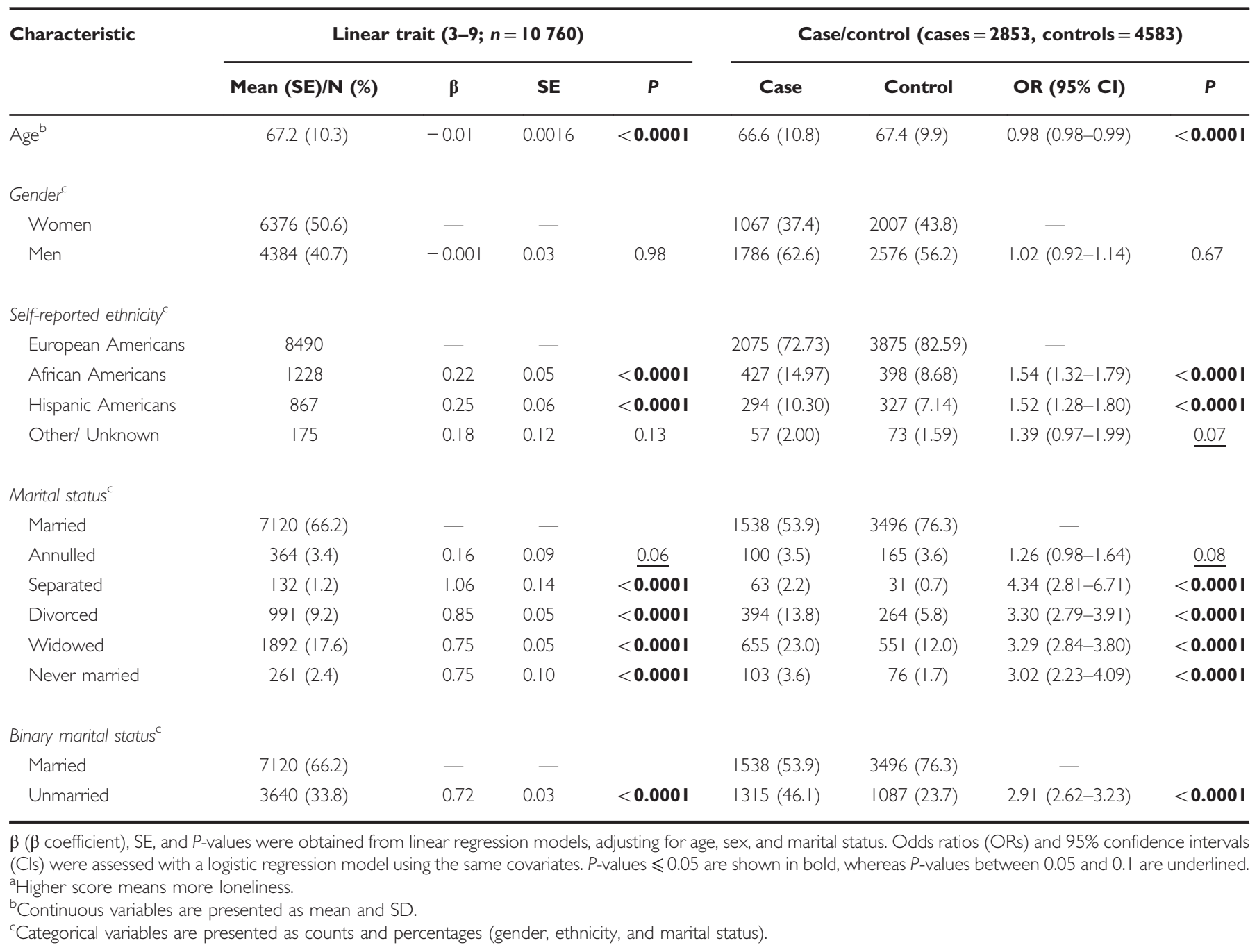

significantly positive for all three neuroticism data sets (see Figure 2, $r_{\mathrm{g}}=0.39, p=4.1 \times 10^{-4} ; r_{\mathrm{g}}=0.40, p=8.4 \times 10^{-5}$; $r_{\mathrm{g}}=0.41, \quad p=2.5 \times 10^{-3}$, respectively) and negative for extraversion $\left(r_{\mathrm{g}}=-0.34, p=0.013\right)$. Unlike the modest evidence from the PRS analysis, schizophrenia and bipolar disorder did not show any significant results. Whereas there was absolutely no evidence for coheritability with major depression in the PRS analysis, there was a trend toward a positive correlation in the LDSC analysis $\left(r_{\mathrm{g}}=0.25, p=0.08\right)$. Similar to the PRS analysis, the 'depressive symptoms' trait was strongly positively correlated with loneliness $\left(r_{\mathrm{g}}=0.39\right.$, $\left.p=2.9 \times 10^{-4}\right)$. We also included height as a negative control in the LDSC analysis; as expected, there was no coheritability between loneliness and height $(p>0.05)$.

\section{DISCUSSION}

Traditionally, the emphasis in research on loneliness has been on environmental predictors and determinants. In the past decade, a series of twin studies have provided estimates of the heritability $\left(h^{2}\right)$ of loneliness. Here we report the first GWAS of loneliness. We have produced the first estimates of the chip heritability $\left(h_{\mathrm{g}}^{2}\right)$ of loneliness (Table $\left.3 ; 14-27 \%\right)$ that appear to account for approximately half of the heritability estimated from twin and family studies (37-55\%). We did not identify any genome-wide significant associations (Figure 1 and Supplementary Figures S4-S6), presumably reflecting the very modest contributions of individual variants. Previous studies have reported associations between polymorphisms in a handful of candidate genes and loneliness (Chou, 2010; Chou et al, 2014; Connelly et al, 2014; Lan et al, 2012; Lucht et al, 2009; Terracciano et al, 2010; Tsai et al, 2012; van Roekel et al, 2010, 2011, 2013; Verhagen et al, 2014; Wang et al, 2013); our study did not provide even nominal evidence for replication, despite our much larger sample size (Table 2). Finally, we identified varying levels of evidence for coheritability between personality traits (positive for neuroticism and negative for extraversion) and psychiatric disease traits (negative for schizophrenia and bipolar disorder and positive for depression). This latter result is especially interesting in light of the behavioral research showing that loneliness and psychiatric illness are related in other contexts (Cacioppo et al, 2015c), and provides novel evidence that such associations may reflect genetic as well as environmental influences. 
Table 2 Association between Loneliness Phenotypes and Candidate Gene Associations Reported in Prior Studies

\begin{tabular}{|c|c|c|c|c|c|c|c|c|c|}
\hline \multirow[t]{2}{*}{ Gene } & \multirow[t]{2}{*}{ Chr } & \multirow{2}{*}{$\begin{array}{l}\text { Reported } \\
\text { SNP }\end{array}$} & \multirow[t]{2}{*}{ Function } & \multirow[t]{2}{*}{ Population } & \multirow{2}{*}{$\begin{array}{l}\text { Sample } \\
\text { size }\end{array}$} & \multirow[t]{2}{*}{ References } & \multicolumn{3}{|c|}{$P$ for association (all subjects/EA-only) ${ }^{a}$} \\
\hline & & & & & & & Linear & Multivariate & Case/control \\
\hline$B D N F$ & 11 & rs53576 b & Intron & Dutch & 305 & Connelly et al (20|4) & NA & NA & NA \\
\hline OXTR & 3 & rs $2254298^{b}$ & Intron & UKI Germany & $7723 / 285 / 89$ & Lucht et al (2009) & $0.61 / 0.7 \mid$ & $0.87 / 0.98$ & $0.98 / 0.48$ \\
\hline GRM8 & 7 & rs| 7864092 & Intron & Italy + US & $3972+839$ & Terracciano et al (2010) & $0.77 / 0.77$ & $0.41 / 0.29$ & $0.98 / 0.83$ \\
\hline CHRNA4 & 20 & rs 1044396 & Synonymous & Taiwan & 192 & Tsai et al $(2012$ & $0.75 / 0.95$ & $0.76 / 0.77$ & $0.73 / 0.82$ \\
\hline$I L-I A$ & 2 & rs| 800587 & 5' UTR & Taiwan & 192 & Wang et al (20|3) & $0.33 / 0.55$ & $0.25 / 0.52$ & $0.69 / 0.68$ \\
\hline \multirow[t]{2}{*}{ CRHRI } & \multirow[t]{2}{*}{17} & rs $|87683|$ & Intron & \multirow[t]{2}{*}{ UK } & \multirow[t]{2}{*}{ ।,374 } & \multirow[t]{2}{*}{ Chou et al (20|4) } & $0.28 / 0.09$ & $0.54 / 0.41$ & $0.14 / 0.06$ \\
\hline & & rs242938 & Intron & & & & $0.74 / 0.46$ & $0.87 / 0.74$ & $0.14 / 0.20$ \\
\hline SLC6A4 & 17 & \multicolumn{2}{|c|}{ Insertion/5-HTTLPR } & The Netherlands & 306 & van Roekel et al (20।0) & NA & NA & NA \\
\hline
\end{tabular}

${ }^{a} P$-values are not corrected for multiple comparisons. We used multivariate or logistic regression models (GEMMA) to account for relatedness. Adjustments include sex, age, and marital status. P-values before '" are for all the 10760 participants, whereas those after "' are for 7556 European Americans only. For the gene MTHFR ( $\mathrm{r}$ | 80 I I 33), the direction of effect was opposite to what had been reported previously. ${ }^{b}$ For those candidate SNPs that were not genotyped/imputed in our study, we identified proxy SNPs with $r^{2}>0.8$ that were genotyped or imputed in our study based on HapMap2. When no proxy SNP could be identified we report NA rather than a $p$-value.

Table 3 Chip Heritability Estimates in European Americans (EAs)

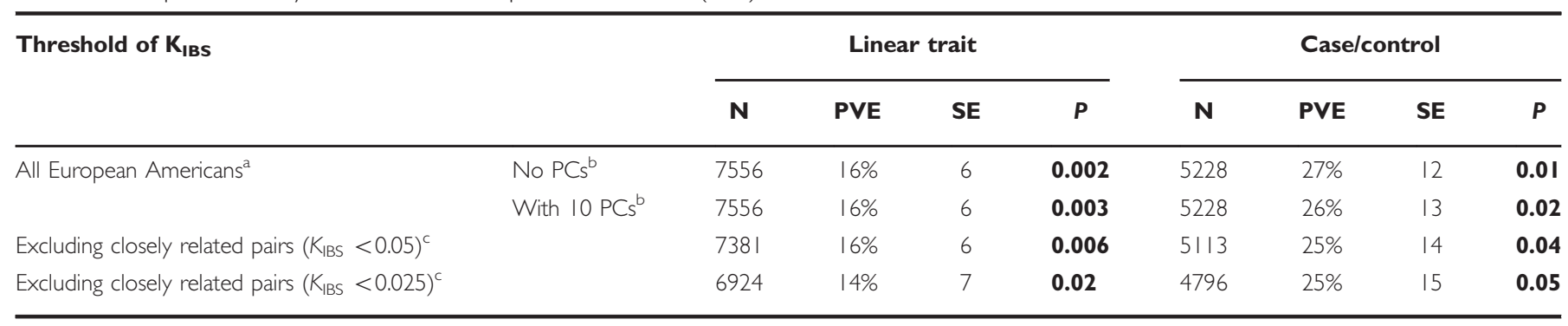

Abbrevations: IBS, identical by descent; PC, principal component; PVE, percent variance explained; SE, standard error.

$P$-values of $\leqslant 0.05$ are in bold.

aUsing the full GRM, $K_{\text {IBS }}$ on all individuals.

${ }^{\text {b} N o ~ P C s: ~ c o v a r i a t e s ~ i n c l u d i n g ~ g e n d e r, ~ a g e ~(c o n t i n u o u s), ~ a n d ~ m a r i t a l ~ s t a t u s ~(b i n a r y) . ~ W i t h ~} 10$ PCs: covariates included the first 10 PCs of genotype data.

${ }^{c}$ The GRM includes only distantly related pairs ( $K_{\mathrm{IBS}}<0.05$ or 0.025$)$. One individual from each relative pair was excluded.

Prior behavioral genetic studies have used adoption designs (McGuire and Clifford, 2000), twin designs (Boomsma et al, 2005; Waaktaar and Torgersen, 2012), a family-based design including nontwin siblings (Boomsma et al, 2006), and an extended twin designs to include the partners and parents of twins (Distel et al, 2010) to estimate the heritability of loneliness in a variety of populations (Goossens et al, 2015). The heritability estimates across these various designs have ranged from 37 to $55 \%$. These estimates reflect the contributions of both common and rare variants. In contrast, estimates of chip heritability only capture the additive contributions of common variation (Yang et al, 2013), and are therefore expected to be lower. As such, they provide insight into the genetic architecture of loneliness, namely that it is polygenic in nature and is likely to be influenced by many common genetic variants of small effect. Our estimates of chip heritability add to existing heritability estimates and also reinforce the notion that both genetic and environmental factors influence loneliness. Future studies might increase heritability by utilizing more environmentally homogeneous populations or by including more environmental variables as covariates.

Our study did not identify any genome-wide significant associations. Although the sample size of slightly $>10000$ individuals provides appreciably greater statistical power than had been available previously, numerous disease and nondisease phenotypes that are known to be heritable have also yielded negative results with similar sample sizes 
Table 4 Associations between Polygenic Scores for Neuroticism from SSGAC (Okbay et al, 20 I 6) and Loneliness in Health and Retirement Study $(H R S)^{a}$

\begin{tabular}{|c|c|c|c|c|c|c|c|c|c|c|c|c|c|c|}
\hline \multirow{3}{*}{$\begin{array}{l}P \text {-value } \\
\text { threshold }\end{array}$} & \multirow{3}{*}{$\begin{array}{c}\text { Num. } \\
\text { of } \\
\text { SNPs }\end{array}$} & \multicolumn{3}{|c|}{ Linear trait ${ }^{\mathbf{b}}$} & \multicolumn{7}{|c|}{ Multivariate traits $^{c}$} & \multicolumn{3}{|c|}{ Case/control $^{d}$} \\
\hline & & \multirow[t]{2}{*}{$\beta$} & \multirow[t]{2}{*}{ SE } & \multirow[t]{2}{*}{$\mathbf{P}$} & \multicolumn{2}{|c|}{ QI:companion } & \multicolumn{2}{|c|}{ Q2: left out } & \multicolumn{2}{|c|}{ Q3: isolated } & \multirow{2}{*}{$\begin{array}{l}P \text { for } \\
\text { overall }\end{array}$} & \multirow[t]{2}{*}{ OR } & \multirow[t]{2}{*}{$95 \% \mathrm{Cl}$} & \multirow[t]{2}{*}{$P$} \\
\hline & & & & & $\beta I$ & $\mathbf{P I}$ & $\beta 2$ & $\mathbf{P 2}$ & $\beta 3$ & P3 & & & & \\
\hline $1 \times 10^{-5}$ & 33 & 0.03 & 0.02 & $\underline{0.08}$ & 0.01 & 0.14 & 0.01 & 0.12 & 0.01 & 0.14 & 0.37 & 1.08 & $1.01-1.14$ & 0.02 \\
\hline $1 \times 10^{-3}$ & 515 & 0.04 & 0.02 & 0.02 & 0.01 & 0.07 & 0.01 & 0.03 & 0.01 & 0.07 & 0.16 & 1.08 & $1.01-1.15$ & 0.02 \\
\hline 0.01 & 3223 & 0.07 & 0.02 & $9.1 \times 10^{-5}$ & 0.02 & 0.001 & 0.03 & 0.0001 & 0.02 & 0.003 & 0.001 & 1.15 & $1.08-1.22$ & $1.2 \times 10^{-5}$ \\
\hline 0.05 & 13036 & 0.08 & 0.02 & $9.3 \times 10^{-6}$ & 0.03 & $3.5 \times 10^{-4}$ & 0.03 & $9.9 \times 10^{-6}$ & 0.02 & 0.0008 & $9.9 \times 10^{-5}$ & 1.17 & $1.10-1.24$ & $1.5 \times 10^{-6}$ \\
\hline 0.1 & 24251 & 0.09 & 0.02 & $4.1 \times 10^{-6}$ & 0.03 & $4.1 \times 10^{-5}$ & 0.03 & $2.9 \times 10^{-5}$ & 0.02 & 0.0005 & $\mathbf{6 . 7} \times 10^{-5}$ & 1.17 & $1.10-1.24$ & $1.4 \times 10^{-6}$ \\
\hline 0.3 & 65722 & 0.09 & 0.02 & $4.1 \times 10^{-7}$ & 0.03 & $3.0 \times 10^{-5}$ & 0.03 & $3.9 \times 10^{-6}$ & 0.03 & $3.3 \times 10^{-5}$ & $1.0 \times 10^{-5}$ & 1.17 & $1.10-1.25$ & $3.6 \times 10^{-7}$ \\
\hline
\end{tabular}

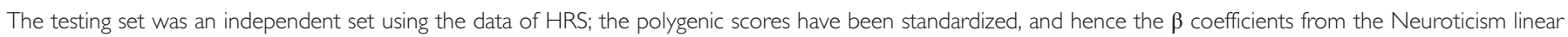
regression model correspond to a I SD change in the polygenic score. $P$-values $\leqslant 0.05$ are in bold, whereas $0.05<P$-values $\leqslant 0$.I are underlined.

aThe polygenic model was developed using SNPs with p-values below the indicated threshold from Neuroticism obtained from Social Science Genetic Association Consortium (SSGAC, Neuroticism_Full.txt).

'Using linear regression model for 6924 unrelated EAs; adjustments included sex, age, and marital status; further adjusting for the top 3 PCs had little impact.

'Using multivariate regression model for 6924 unrelated EAs, same adjustments as above.

Using logistic regression model for 4796 unrelated EAs (cases/controls = 1632: 31 64), same adjustments as above.

(see, eg, Major Depressive Disorder Working Group of the Psychiatric GWAS Consortium et al, 2013).

Our study provided an efficient means of testing previously reported associations between SNPs in candidate genes and loneliness. Despite an active literature in this area, we did not find support for any of the previously reported candidate gene associations. This is consistent with our previous experience with candidate gene-based studies (Hart et al, 2013). Several of the previously published candidate gene studies reported effect sizes that are much larger than those typically observed in genome-wide association studies that in hindsight should have generated more skepticism about those results. Although our findings cast doubt on the previously reported associations-or at least on the original effect sizes that were identified-there are potentially important differences between our study design and the previously published candidate gene studies. For instance, our population was based in the United States and was made up of older adults, many of whom were in stable longterm relationships, whereas approximately half of the candidate gene studies utilized samples of adolescents from the Netherlands or Germany (Lucht et al, 2009; van Roekel et al, 2010, 2011, 2013; Verhagen et al, 2014). Therefore, although our study benefited from a larger sample size than any of the previously reported candidate gene studies, we cannot discount the possibility that differences in the methodologies or the population under study led to our failure to replicate the previously published results. The phenotyping procedure used in the current study has been found to correlate highly $(r=0.88)$ with a more in-depth phenotype for loneliness (Hughes et al, 2004), and the candidate gene studies using older adults from the United Kingdom and Taiwan provided no greater evidence for replication than the studies using adolescents (Chou, 2010,
2014; Connelly et al, 2014; Lan et al, 2012; Tsai et al, 2012; Wang et al, 2013).

We observed strong genetic correlations between loneliness and two personality dimensions: neuroticism and extraversion (Table 4, Figure 2, and Supplementary Tables S3-S5). The direction of these effects was consistent with the correlations identified previously: greater loneliness has been shown to be positively correlated with neuroticism and negatively correlated with extraversion (see, eg, Cacioppo et al, 2006; Mund and Neyer, 2015). Neuroticism is characterized by high negative affectivity, a characteristic also seen in loneliness. Although prior research has shown loneliness and neuroticism to be stochastically and functionally separable, the results from the current study suggest there may be a shared genetic predisposition that contributes to both phenotypes. The multivariate PRS analyses provide information regarding the coheritability between loneliness and extraversion. The results show that whereas all three questions contributed to the genetic correlation with neuroticism (Table 4 and Supplementary Tables S3 and S4), only the items regarding lack of companionship and feeling left out contributed to the genetic correlation with extraversion (Supplementary Tables S5).

We see our study as being a part of an important trend that attempts to relate the genetic causes of psychiatric disease diagnoses to continuously variable traits that represent heritable personality characteristics. It is widely accepted that humans have varying degrees of sensitivity to social isolation; however, the question of whether or not the genetic basis of this variability also underlies the risk for common psychiatric diseases remains largely unexplored. We have previously reported that genetic variation in the initial sensitivity to the euphoric effects of amphetamine is genetically correlated with the risk for both schizophrenia and ADHD (Hart et al, 2014). That provocative finding 


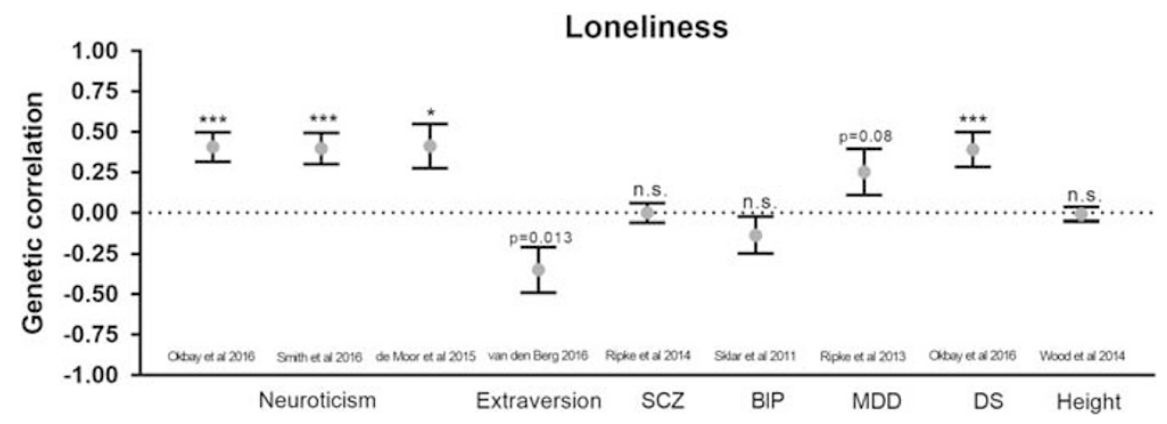

Figure 2 Genetic correlations between loneliness (EA-only, case/control) and nine additional traits: personality traits (neuroticism, extraversion), psychiatric conditions (schizophrenia (SCZ), bipolar disorder (BP), and major depression disorder (MDD)), a depressive symptoms scale (DS), and height. Error bars represent SE. $* P<0.01$, **** $p<0.0001$, NS $p>0.05$.

provides an example of using genetic variation in a nondisease trait to obtain novel insights into the genetic basis of psychiatric diseases. In the present study, we explored whether or not genetic risk for loneliness mapped onto genetic risk for major psychiatric diseases. Our results provided limited support for this hypothesis (Figure 2 and Supplementary Tables S6-S9). The linear phenotype did not show any evidence for coheritability with schizophrenia, bipolar disorder, or major depression. However, when we used a multitrait mapping approach, which allowed us to consider each of the three questionnaire items independently, we saw suggestive evidence for coheritability between the second question ('how often do you feel left out?') and both schizophrenia and bipolar disorder. The relationship between loneliness and these two diseases was very weakly negative, meaning that being lonelier is associated with reduced risk of disease. We also used summary statistics from the case/control GWAS to perform LDSC that did not support coheritability with schizophrenia or bipolar disorder, but did show a trend toward a positive genetic correlation with major depression $(p=0.08$; lonelier was associated with greater risk for depression). The nonpsychiatric trait termed 'depressive symptoms' was more robustly positively correlated with loneliness in both the PRS and the LDSC analyses (lonelier was genetically correlated with more depressive symptoms). Because of the number of tests performed and the modest levels of significance for the psychiatric diseases, those results should be considered suggestive until they are replicated. Although we assume that few participants in the HRS study would be diagnosed with schizophrenia, such data were not available; therefore, we cannot exclude the possibility that our findings are secondary to the effects of schizophrenia on self-reported loneliness. The direction of the effect suggests that greater genetic risk for loneliness is negatively associated with these psychiatric diseases. We have previously hypothesized that loneliness reflects an adaptive drive toward social interaction that is consistent with the direction of the observed correlation. Future studies of loneliness and other social behavior traits may continue to inform our understanding of the role of social behavior in psychiatric health and disease (Cacioppo et al, 2014).

In summary, we have performed the first GWAS of loneliness. Our study has identified significant evidence for heritability, but did not identify specific loci associated with loneliness nor was it able to replicate previously reported candidate gene associations. Finally, we identified strong evidence for coheritability between loneliness and neuroticism, extraversion, and 'depressive symptoms', and suggestive evidence for coheritability between loneliness and schizophrenia, bipolar disorder, and major depressive disorder. We believe that future studies of loneliness, as well as additional studies of other social neuroscience phenotypes, may continue to enrich our understanding of the ways in which our genetic inheritance fundamentally influences individual and social behavior.

\section{FUNDING AND DISCLOSURE}

The authors declare no conflict of interest.

\section{ACKNOWLEDGMENTS}

We thank Hae Kyung Im for helpful conversations during the preparation of this manuscript. Research reported in this publication was supported by the National Institute on Aging of the National Institutes of Health under award number R37-AG033590 and by the National Center for the National Center of Advancing Translational Sciences of the National Institutes of Health under award number KL2TR000431 (LKD). The content is solely the responsibility of the authors and does not necessarily represent the official views of the National Institutes of Health.

\section{REFERENCES}

Adam EK, Hawkley LC, Kudielka BM, Cacioppo JT (2006). Day-today dynamics of experience-cortisol associations in a populationbased sample of older adults. Proc Natl Acad Sci USA 103: $17058-17063$.

Bartels M, Cacioppo JT, Hudziak JJ, Boomsma DI (2008). Genetic and environmental contributions to stability in loneliness throughout childhood. Am J Med Genet B Neuropsychiatr Genet 147: 385-391.

Baumeister R, DeWall N (2005). The inner dimension of social exclusion: intelligent thought and self-regulation among rejected persons. In Williams K, Forgas J, Von Hippel W(eds). The Social Outcast: Ostracism, Social Exclusion, Rejection, and Bullying. Psychology Press: New York. pp 53-76. 
Boomsma DI, Cacioppo JT, Muthen B, Asparouhov T, Clark S (2007). Longitudinal genetic analysis for loneliness in Dutch twins. Twin Res Hum Genet 10: 267-273.

Boomsma DI, Cacioppo JT, Slagboom PE, Posthuma D (2006). Genetic linkage and association analysis for loneliness in Dutch twin and sibling pairs points to a region on chromosome 12q2324. Behav Genet 36: 137-146.

Boomsma DI, Willemsen G, Dolan CV, Hawkley LC, Cacioppo JT (2005). Genetic and environmental contributions to loneliness in adults: the Netherlands twin register study. Behav Genet 35: $745-752$.

Booth R (2000). Loneliness as a component of psychiatric disorders. Medscape Gen Med 2: 1-7.

Bulik-Sullivan B, Finucane HK, Anttila V, Gusev A, Day FR, Loh P-R, ReproGen Consortium, Psychiatric Genomics Consortium, Genetic Consortium for Anorexia Nervosa of the Wellcome Trust Case Control Consortium 3 et al (2015a). An atlas of genetic correlations across human diseases and traits. Nat Genet 47: 1236-1241.

Bulik-Sullivan BK, Loh P-R, Finucane HK, Ripke S, Yang J, Patterson $\mathrm{N}$ et al (2015b). LD Score regression distinguishes confounding from polygenicity in genome-wide association studies. Nat Genet 47: 291-295.

Cacioppo JT, Cacioppo S, Boomsma DI (2014). Evolutionary mechanisms for loneliness. Cogn Emot 28: 3-21.

Cacioppo JT, Cacioppo S, Capitanio JP, Cole SW (2015a). The neuroendocrinology of social isolation. Annu Rev Psychol 66: 733-767.

Cacioppo JT, Ernst JM, Burleson MH, McClintock MK, Malarkey WB, Hawkley LC et al (2000). Lonely traits and concomitant physiological processes: the MacArthur social neuroscience studies. Int J Psychophysiol 35: 143-154.

Cacioppo JT, Hawkley LC, Berntson GG, Ernst JM, Gibbs AC, Stickgold R et al (2002). Do lonely days invade the nights? Potential social modulation of sleep efficiency. Psychol Sci 13: 384-387.

Cacioppo JT, Hawkley LC, Thisted RA (2010). Perceived social isolation makes me sad: 5-year cross-lagged analyses of loneliness and depressive symptomatology in the Chicago Health, Aging, and Social Relations Study. Psychol Aging 25: 453-463.

Cacioppo JT, Hughes ME, Waite LJ, Hawkley LC, Thisted RA (2006). Loneliness as a specific risk factor for depressive symptoms: cross-sectional and longitudinal analyses. Psychol Aging 21: $140-151$.

Cacioppo S, Balogh S, Cacioppo JT (2015b). Implicit attention to negative social, in contrast to nonsocial, words in the Stroop task differs between individuals high and low in loneliness: Evidence from event-related brain microstates. Cortex 70: 213-233.

Cacioppo S, Grippo AJ, London S, Goossens L, Cacioppo JT (2015c). Loneliness: clinical import and interventions. Perspect Psychol Sci 10: 238-249.

Cheng R, Parker CC, Abney M, Palmer AA (2013). Practical considerations regarding the use of genotype and pedigree data to model relatedness in the context of genome-wide association studies. G3 (Bethesda) 3: 1861-1867.

Chou KL (2010). Moderating effect of apolipoprotein genotype on loneliness leading to depressive symptoms in Chinese older adults. Am J Geriatr Psychiatry 18: 313-322.

Chou KL, Cacioppo JT, Kumari M, Song YQ (2014). Influence of social environment on loneliness in older adults: Moderation by polymorphism in the CRHR1. Am J Geriatr Psychiatry 22: 510-518.

Cole SW, Hawkley LC, Arevalo JM, Cacioppo JT (2011). Transcript origin analysis identifies antigen-presenting cells as primary targets of socially regulated gene expression in leukocytes. Proc Natl Acad Sci USA 108: 3080-3085.

Cole SW, Hawkley LC, Arevalo JM, Sung CY, Rose RM, Cacioppo JT (2007). Social regulation of gene expression in human leukocytes. Genome Biol 8: R189.

Connelly JJ, Golding J, Gregory SP, Ring SM, Davis JM, Davey Smith G et al (2014). Personality, behavior and environmental features associated with OXTR genetic variants in British mothers. PLoS One 9: e90465.

Coyle CE, Dugan E (2012). Social isolation, loneliness and health among older adults. J Aging Health 24: 1346-1363.

Distel MA, Willemsen G, Ligthart L, Derom CA, Martin NG, Neale MC et al (2010). Genetic covariance structure of the four main features of borderline personality disorder. J Pers Disord 24: 427-444.

Dixon D, Cruess S, Kilbourn K, Klimas N, Fletcher MA, Ironson G et al (2006). Social Support Mediates Loneliness and Human Herpesvirus Type 6 (HHV-6) Antibody Titers. J Appl Soc Psychol 31: 1111-1132.

Doane LD, Adam EK (2010). Loneliness and cortisol: momentary, day-to-day, and trait associations. Psychoneuroendocrinology 35: $430-441$.

Finucane HK, Bulik-Sullivan B, Gusev A, Trynka G, Reshef Y, Loh PR et al (2015). Partitioning heritability by functional annotation using genome-wide association summary statistics. Nat Genet 47: 1228-1235.

Glaser K, Evandrou M, Tomassini C (2005). The health consequences of multiple roles at older ages in the UK. Health Soc Care Community 13: 470-477.

Glaser R, Kiecolt-Glaser JK, Speicher CE, Holliday JE (1985). Stress, loneliness, and changes in herpesvirus latency. J Behav Med 8: 249-260.

Goossens L, van Roekel E, Verhagen M, Cacioppo JT, Cacioppo S, Maes $M$ et al (2015). The genetics of loneliness: linking evolutionary theory to genome-wide genetics, epigenetics, and social science. Perspect Psychol Sci 10: 213-226.

Hackett RA, Hamer M, Endrighi R, Brydon L, Steptoe A (2012). Loneliness and stress-related inflammatory and neuroendocrine responses in older men and women. Psychoneuroendocrinology 37: 1801-1809.

Hart AB, de Wit H, Palmer AA (2013). Candidate gene studies of a promising intermediate phenotype: failure to replicate. Neuropsychopharmacology 38: 802-816.

Hart AB, Gamazon ER, Engelhardt BE, Sklar P, Kahler AK, Hultman CM et al (2014). Genetic variation associated with euphorigenic effects of $\mathrm{d}$-amphetamine is associated with diminished risk for schizophrenia and attention deficit hyperactivity disorder. Proc Natl Acad Sci USA 111: 5968-5973.

Hawkley LC, Hughes ME, Waite LJ, Masi CM, Thisted RA, Cacioppo JT (2008). From social structural factors to perceptions of relationship quality and loneliness: The Chicago health, aging, and social relations study. J Gerontol B Psychol Sci Soc Sci 63B: S375-S384.

Hawkley LC, Masi CM, Berry JD, Cacioppo JT (2006). Loneliness is a unique predictor of age-related differences in systolic blood pressure. Psychol Aging 21: 152-164.

Hawkley LC, Preacher KJ, Cacioppo JT (2010a). Loneliness impairs daytime functioning but not sleep duration. Health Psychol 29: 124-129.

Hawkley LC, Thisted RA, Cacioppo JT (2009). Loneliness predicts reduced physical activity: cross-sectional \& longitudinal analyses. Health Psychol 28: 354-363.

Hawkley LC, Thisted RA, Masi CM, Cacioppo JT (2010b). Loneliness predicts increased blood pressure: 5-year cross-lagged analyses in middle-aged and older adults. Psychol Aging 25: 132-141.

Health_and_Retirement_Study (2012). Core public use dataset. Produced and distributed by the University of Michigan with funding from the National Institute on Aging (grant number NIA U01AG009740). Ann Arbor, MI.

Holt-Lunstad J, Smith TB, Baker M, Harris T, Stephenson D (2015). Loneliness and social isolation as risk factors for mortality: a meta-analytic review. Perspect Psychol Sci 10: 227-237.

Hughes ME, Waite LJ, Hawkley LC, Cacioppo JT (2004). A short scale for measuring loneliness in large surveys: results from two population-based studies. Res Aging 26: 655-672. 
International HapMap Consortium (2010). Integrating common and rare genetic variation in diverse human populations. Nature 467: $52-58$

Jacobs JM, Cohen A, Hammerman-Rozenberg R, Stessman J (2006). Global sleep satisfaction of older people: the Jerusalem Cohort Study. J Am Geriatr Soc 54: 325-329.

Jaremka LM, Fagundes CP, Peng J, Bennett JM, Glaser R, Malarkey WB et al (2013). Loneliness promotes inflammation during acute stress. Psychol Sci 24: 1089-1097.

Kiecolt-Glaser JK, Ricker D, George J, Messick G, Speicher CE, Garner W et al (1984). Urinary cortisol levels, cellular immunocompetency, and loneliness in psychiatric inpatients. Psychosom Med 46: 15-23.

Kong F, You X (2013). Loneliness and self-esteem as mediators between social support and life satisfaction in late adolescence. Soc Indic Res 110: 271-279.

Kurina LM, Knutson KL, Hawkley LC, Cacioppo JT, Lauderdale DS, Ober C (2011). Loneliness is associated with sleep fragmentation in a communal society. Sleep 34: 1519-1526.

Lan WH, Yang AC, Hwang JP, Hong CJ, Liou YJ, Yeh HL et al (2012). Association of MTHFR C677T polymorphism with loneliness but not depression in cognitively normal elderly males. Neurosci Lett 521: 88-91.

Lucht MJ, Barnow S, Sonnenfeld C, Rosenberger A, Grabe HJ, Schroeder W et al (2009). Associations between the oxytocin receptor gene (OXTR) and affect, loneliness and intelligence in normal subjects. Prog Neuropsychopharmacol Biol Psychiatry 33: 860-866.

Luo Y, Hawkley LC, Waite LJ, Cacioppo JT (2012). Loneliness, health, and mortality in old age: a national longitudinal study. Soc Sci Med 74: 907-914.

Major Depressive Disorder Working Group of the Psychiatric GWAS Consortium, Ripke S, Wray NR, Lewis CM, Hamilton SP, Weissman MM et al (2013). A mega-analysis of genome-wide association studies for major depressive disorder. Mol Psychiatry 18: $497-511$.

McGuire S, Clifford J (2000). Genetic and environmental contributions to loneliness in children. Psychol Sci 11: 487-491.

Mund M, Neyer FJ (2015). The winding paths of the lonesome cowboy: evidence for mutual influences between personality, subjective health, and loneliness. J Pers 84: 646-657.

Okbay A, Baselmans BML, De Neve J-E, Turley P, Nivard MG, Fontana MA et al. (2016). Genetic variants associated with subjective well-being, depressive symptoms, and neuroticism identified through genome-wide analyses. Nat Genet 48: 624-633.

Pressman SD, Cohen S, Miller GE, Barkin A, Rabin BS, Treanor JJ (2005). Loneliness, social network size, and immune response to influenza vaccination in college freshmen. Health Psychol 24: 297-306.

Purcell S, Neale B, Todd-Brown K, Thomas L, Ferreira MA, Bender D et al (2007). PLINK: a tool set for whole-genome association and population-based linkage analyses. Am J Hum Genet 81: 559-575.

Russell D (1996). UCLA Loneliness Scale (Version 3): Reliability, validity, and factor structure. J Pers Asses 66: 20-40.

Russell D, Peplau LA, Cutrona CE (1980). The Revised UCLA Loneliness Scale: Concurrent and discriminate validity evidence. J Personality Soc Psychol 39: 472-480.

Steptoe A, Owen N, Kunz-Ebrecht SR, Brydon L (2004). Loneliness and neuroendocrine, cardiovascular, and inflammatory stress responses in middle-aged men and women. Psychoneuroendocrinology 29: 593-611.
Straits-Tröster KA, Patterson TL, Semple SJ, Temoshok L, Roth PG, McCutchan JA et al (1994). The relationship between loneliness, interpersonal competence, and immunologic status in HIVinfected men. Psychol Health 9: 205-219.

Terracciano A, Tanaka T, Sutin AR, Sanna S, Deiana B, Lai S et al (2010). Genome-wide association scan of trait depression. Biol Psychiatry 68: 811-817.

Tsai SJ, Yeh HL, Hong CJ, Liou YJ, Yang AC, Liu ME et al (2012). Association of CHRNA4 polymorphism with depression and loneliness in elderly males. Genes Brain Behav 11: 230-234.

van Roekel E, Goossens L, Scholte RH, Engels RC, Verhagen M (2011). The dopamine D2 receptor gene, perceived parental support, and adolescent loneliness: longitudinal evidence for gene-environment interactions. J Child Psychol Psychiatry 52: 1044-1051.

van Roekel E, Scholte RH, Verhagen M, Goossens L, Engels RC (2010). Loneliness in adolescence: gene $\mathrm{x}$ environment interactions involving the serotonin transporter gene. J Child Psychol Psychiatry 51: 747-754.

van Roekel E, Verhagen M, Scholte RH, Kleinjan M, Goossens L, Engels RC (2013). The oxytocin receptor gene (OXTR) in relation to state levels of loneliness in adolescence: evidence for microlevel gene-environment interactions. PLoS One 8: e77689.

VanderWeele TJ, Hawkley LC, Cacioppo JT (2012). On the reciprocal association between loneliness and subjective wellbeing. Am J Epidemiol 176: 777-784.

VanderWeele TJ, Hawkley LC, Thisted RA, Cacioppo JT (2011). A marginal structural model analysis for loneliness: implications for intervention trials and clinical practice. J Consult Clin Psychol 79: 225-235.

Verhagen M, van Roekel E, Engels RC (2014). Involvement of the BDNF gene in loneliness in adolescence: a report of opposite gene effects in boys and girls. PLoS One 9: e92768.

Waaktaar T, Torgersen S (2012). Genetic and environmental causes of variation in perceived loneliness in young people. Am J Med Genet B Neuropsychiatr Genet 159B: 580-588.

Wang EH, Hong CJ, Yeh HL, Liou YJ, Yang AC, Liu ME et al (2013). Interleukin-1 alpha (rs1800587) genetic polymorphism is associated with specific cognitive functions but not depression or loneliness in elderly males without dementia. Neurosci Lett $\mathbf{5 5 6}$ 69-72.

Whisman MA (2010). Loneliness and the metabolic syndrome in a population-based sample of middle-aged and older adults. Health Psychol 29: 550-554.

Wilson RS, Krueger KR, Arnold SE, Schneider JA, Kelly JF, Barnes LL et al (2007). Loneliness and risk of Alzheimer disease. Arch Gen Psychiatry 64: 234-240.

Yang J, Benyamin B, McEvoy BP, Gordon S, Henders AK, Nyholt DR et al (2010). Common SNPs explain a large proportion of the heritability for human height. Nat Genet 42: 565-569.

Yang J, Lee SH, Goddard ME, Visscher PM (2011). GCTA: a tool for genome-wide complex trait analysis. Am J Hum Genet 88: 76-82.

Yang J, Lee SH, Goddard ME, Visscher PM (2013). Genome-wide complex trait analysis (GCTA): methods, data analyses, and interpretations. Methods Mol Biol 1019: 215-236.

Yang J, Zaitlen NA, Goddard ME, Visscher PM, Price AL (2014). Advantages and pitfalls in the application of mixed-model association methods. Nat Genet 46: 100-106.

Zhou X, Stephens M (2012). Genome-wide efficient mixed-model analysis for association studies. Nat Genet 44: 821-824.

Supplementary Information accompanies the paper on the Neuropsychopharmacology website (http://www.nature.com/npp) 